\title{
Evaluation of Aldose Reductase, Protein Glycation, and Antioxidant Inhibitory Activities of Bioactive Flavonoids in Matricaria recutita L. and Their Structure-Activity Relationship
}

\author{
Seung Hwan Hwang, ${ }^{1}$ Zhiqiang Wang, ${ }^{1,2}$ Yanymee N. Guillen Quispe, ${ }^{1}$ Soon Sung Lim $\left(\mathbb{D},{ }^{1,3,4}\right.$ \\ and Jae Myung Yu $\mathbb{D}^{5}$ \\ ${ }^{1}$ Department of Food Science and Nutrition, Hallym University, 1 Hallymdeahak-gil, Chuncheon, Republic of Korea \\ ${ }^{2}$ College of Public Health, Hebei University, Baoding 071002, China \\ ${ }^{3}$ Institute of Korean Nutrition, Hallym University, 1 Hallymdeahak-gil, Chuncheon, Republic of Korea \\ ${ }^{4}$ Institute of Natural Medicine, Hallym University, 1 Hallymdeahak-gil, Chuncheon, Republic of Korea \\ ${ }^{5}$ Hallym University Kangnam Sacred Heart Hospital, 1 Singil-ro, Yeoungdeungpo-gu, Seoul, Republic of Korea
}

Correspondence should be addressed to Jae Myung Yu; jaemyungyu@hotmail.com

Received 13 November 2017; Accepted 4 January 2018; Published 10 April 2018

Academic Editor: Hiroshi Okamoto

Copyright $\odot 2018$ Seung Hwan Hwang et al. This is an open access article distributed under the Creative Commons Attribution License, which permits unrestricted use, distribution, and reproduction in any medium, provided the original work is properly cited.

\begin{abstract}
The inhibitory activities of Matricaria recutita L. 70\% methanol extract were evaluated by isolating and testing 10 of its compounds on rat lens aldose reductase (RLAR), advanced glycation end products (AGEs), and 2,2-diphenyl-1-picrylhydrazyl (DPPH) radical scavenging. Among these compounds, apigenin-7-O- $\beta$-D-glucoside, luteolin-7-O- $\beta$-D-glucoside, apigenin-7-O- $\beta$-D-glucuronide, luteolin-7-O- $\beta$-D-glucuronide, 3,5-O-di-caffeoylquinic acid, apigenin, and luteolin showed potent inhibition, and their $\mathrm{IC}_{50}$ values in RLAR were $4.25,1.12,1.16,0.85,0.72,1.72$, and $1.42 \mu \mathrm{M}$, respectively. Furthermore, these compounds suppressed sorbitol accumulation in rat lens under high-glucose conditions, demonstrating their potential to prevent sorbitol accumulation ex vivo. Notably, luteolin-7-O- $\beta$-D-glucuronide and luteolin showed antioxidative as well as AGE-inhibitory activities (IC I0 $_{50}$ values of these compounds in AGEs were 3.39 and $6.01 \mu \mathrm{M}$ ). These results suggest that the M. recutita extract and its constituents may be promising agents for use in the prevention or treatment of diabetic complications.
\end{abstract}

\section{Introduction}

Persistent hyperglycemia induces abnormal changes, such as increased formation of advanced glycation end products (AGEs) and polyol pathway flux, and the overactivation of protein kinase C isoforms [1]. Diabetic complications including neuropathy, nephropathy, cataracts, and retinopathy are considered to be caused by the accumulation of sorbitol, which is produced from glucose by aldose reductase in the polyol pathway [2]. Aldose reductase (AR, EC 1.1.1.21) catalyzes the reduction of glucose to the corresponding sugar alcohol, sorbitol, which is subsequently metabolized to fructose by sorbitol dehydrogenase [3]. AR is present in almost all mammalian cells, especially in lens, retina and sciatic nerves, which are thus affected by diabetic complications
[4]. Increased polyol pathway flux leads to the accumulation of sorbitol in the lens fiber, thus causing an influx of water, generation of osmotic stress, and cataract formation [5].

Reducing sugars can react nonenzymatically with the amino groups of proteins to form reversible Schiff bases. These early glycation products undergo further complex reactions such as rearrangement, dehydration, and condensation to become irreversibly cross-linked, fluorescent derivatives termed AGEs [6]. The formation and accumulation of AGEs in various tissues have been reported to progress at an accelerated rate under hyperglycemic conditions with oxidative stress [7]. This induces oxidative stress and has deleterious effects on various cellular functions Therefore, protein glycation reactions leading to AGEs are thought to be a major cause of different diabetic complications and inhibition of 
AGE formation could be a novel therapeutic target to prevent complications in diabetes [8].

Flavonoids obtained from natural extracts were reported to have strong AR inhibitory activity and may improve symptoms associated with diabetic complications $[9,10]$. In addition, many flavonoid and phenol constituents isolated from natural extracts have various biological activities, including neuroprotective effects in diabetic complications, as well as anti-inflammatory, antidiabetic, and renal protective effects [11-13]. These are distinguished by the number and arrangement of their functional groups and glycosylation. The number of known flavonoids is greater than 4000, and their inhibitory activities are highly varied according to the position and number of functional groups, as well as the glycosylation patterns on flavonoid aglycone [14].

Matricaria recutita L. (MR) belongs to the Asteraceae (Compositae) family, one of the largest plant families $(23,600$ species $)$, and is a herbaceous plant that is indigenous to Europe and Western Asia [15]. MR is a traditional Chinese medicinal herb that has been used in China for centuries to treat various diseases including digestive system illness and diarrhea [16]. The recent work of many research team reported that MR extract also showed antiallergic, antiinflammatory, and anticancer properties [17]. In addition, MR is a source of phenolic compounds, namely, the flavonoids apigenin, quercetin, kaempferol, and luteolin, as well as their glucosides and also coumarins, which are considered to be the major bioactive compounds of chamomile $[18,19]$. Recently studies reported that MR dosedependently decreased the intestinal absorption of glucose, and in vitro, MR treatment showed significant protective effects (liver, kidney, and lipid metabolic parameters) for high fat diet-induced obesity and lipotoxicity in rats [20]. In addition, two new acylated apigenin glucosides that were identified as apigenin-7-O-(4" -malonyl $)-\beta$-D-glucopyranoside and apigenin-7-O-( $4^{\prime \prime}$-malonyl- $6^{\prime \prime}$-acetyl $)-\beta$-D-glucopyranoside were isolated from edge flowers of Matricaria chamomilla [21].

To date, no data have been published on the inhibitory effects of MR extract and its constituents on rat lens $A R$ (RLAR), AGEs, and 2,2-diphenyl-1-picrylhydrazyl (DPPH) radical scavenging regulation. Therefore, the inhibitory activities of compounds isolated from MR on RLAR, AGEs, $\mathrm{DPPH}$, and sorbitol accumulation were investigated to evaluate potential treatments for diabetes-related complications. As well as, we discuss here the structure activity relationships (SAR) of MR extract constituents that could potentially inhibit diabetic complications.

\section{Materials and Methods}

2.1. General. ${ }^{1} \mathrm{H}$ and ${ }^{13} \mathrm{C}$ NMR spectra and correlation NMR spectra such as COSY, HMBC, HMQC, and DEPT were obtained from a Bruker Avance DPX 400 (or 600) spectrometer. These spectra were obtained at operating frequencies of $400 \mathrm{MHz}\left({ }^{1} \mathrm{H}\right)$ and 100 (or 150$) \mathrm{MHz}\left({ }^{13} \mathrm{C}\right.$ ) with $\mathrm{CD}_{3} \mathrm{OD}$, and TMS was used as an internal standard. Chemical shifts were reported in $\delta$ values.
2.2. Chemicals and Reagents. L-Ascorbic acid, DPPH, dimethylsulfoxide (DMSO), nicotinamide adenine dinucleotide phosphate (NADPH), DL-glyceraldehyde dimer, bovine serum albumin, methylglyoxal, quercetin, aminoguanidine, sodium phosphate dibasic anhydrous, sodium dihydrogen phosphate, ammonium sulfate, potassium dihydrogen phosphate, sodium hydroxide, sorbitol, and glucose were purchased from Sigma-Aldrich (St. Louis, MO, USA). Sephadex LH-20 was purchased from Wako GE Heakthcare (Milwaukee, WI, USA). All solvents and $\mathrm{CD}_{3} \mathrm{OD}$ used the analytical grade of Sigma-Aldrich (St. Louis, MO, USA).

2.3. Plant Materials. Dried MR leaves (Asteraceae) was obtained from local markets in the department of La Libertad in Peru in May 2015. A voucher was deposited at the Center for Efficacy Assessment and Development of Functional Foods and Drugs, Hallym University (P2016-MR). The specimen was authenticated by Paul H. Gonzales Arce in Museo de Historia Natural Universidad Nacional Mayor de San Marcos, Lima, Peru.

2.4. Extraction and Isolation. A dried MR leaf (50 g) was extracted with $70 \%$ methanol $(\mathrm{MeOH}, 0.5 \mathrm{~L} \times 2$ times) for $3 \mathrm{~h}$ at room temperature. The combined filtrates were concentrated to dryness in vacuo at $40^{\circ} \mathrm{C}$. The extract showed strong inhibitory effects on RLAR, AGEs, and DPPH radical scavenging activity. This extract ( $3 \mathrm{~g})$ therefore underwent chromatography on a Sephadex LH-20 column using $\mathrm{MeOH}$ as the eluent to obtain 16 pooled fractions (MR-SFrac 1-16). Compounds 1 (3.3 mg), 2, and 3 (5.8 mg each) were obtained directly from MR-SFrac 3, 5, and 7, respectively. MR-SFrac 9 was purified to yield the compound $4(3.3 \mathrm{mg})$ and $5(9.3 \mathrm{mg})$ by recycling HPLC with a gradient system from $20 \%$ to $35 \%$ $\mathrm{MeOH}$. MR-SFrac 10 and 11 were further fractionated by Sephadex LH-20 with $70 \% \mathrm{MeOH}$ to obtain compounds 6 (6.1 mg) and 7 (1.3 mg). MR-SFrac 13-15 were further fractionated by Sephadex LH-20 with acetone to obtain compounds $8(5.5 \mathrm{mg})$ and $9(1.6 \mathrm{mg})$. Compound $10(1.9 \mathrm{mg})$ was isolated via silica-gel column chromatography and eluted with a solvent mixture of methyl chloride and $\mathrm{MeOH}$ (from $20: 0$ to $1: 1, v / v$ ).

2.5. Experimental Animals. Experimental animals used in this study were ten male Sprague-Dawley rats with body weight of 250-280 g purchased from Koatech Inc. (Seoul, Korea). They were adapted to a breeding environment of $23 \pm 1^{\circ} \mathrm{C}$, with $60 \pm 5 \%$ humidity, below 60 phones, less than $20 \mathrm{ppm}$ odor, 150-300 lux illumination, and 12 hour light and shade cycle for one week with sufficient food and water. Experiments with animals, as well as their breeding and management, were conducted in accordance with the Guide for the Care and Use of Laboratory Animals, and experiments were performed with the authorization of the Ethics Committee of Hallym University (Hallym-2016-03). Experiments were performed during the light phase of the cycle (10:00-17:00). The rats were anesthetized prior to the removal of the lense$s$, and the lenses of both eyes were removed from the rats for AR experiment. The length of time between the removal of lenses and euthanasia is $2-5 \mathrm{~min}$ and after the removal of 
lenses, the animals were immediately euthanized by carbon dioxide $\left(\mathrm{CO}_{2}\right)$ inhalation.

2.6. Preparation of RLAR Homogenate. Crude RLAR was prepared as follows: lenses were removed from Sprague-Dawley rats (weighing 250-280 g) and frozen at $-70^{\circ} \mathrm{C}$ until use. Noncataractous transparent lenses were pooled and a homogenate was prepared in $0.1 \mathrm{M}$ phosphate buffered saline ( $\mathrm{pH}$ 6.2). The RLAR homogenate was then centrifuged at $10,000 \mathrm{~g}$ for $20 \mathrm{~min}$ at $4^{\circ} \mathrm{C}$ in a refrigerated centrifuge. The supernatant was collected and used as RLAR [22].

2.7. Determination of RLAR Inhibition. A total of $531 \mu \mathrm{L}$ of $0.1 \mathrm{M}$ potassium buffer ( $\mathrm{pH} 7.0$ ), $90 \mu \mathrm{L}$ of NADPH solution (1.6 $\mathrm{mM}$ in potassium buffer), $90 \mu \mathrm{L}$ of RLAR homogenate (6.5 U/mg), $90 \mu \mathrm{L}$ of ammonium sulfate solution $(4 \mathrm{M}$ in potassium buffer), and $90 \mu \mathrm{L}$ of DL-glyceraldehyde $(25 \mathrm{mM}$ in potassium buffer) were mixed with $9 \mu \mathrm{L}$ of different concentrations of samples $(1-0.1 \mathrm{mg} / \mathrm{mL}$ in DMSO, less than $1 \%$ in total mixture) in a cuvette, and the activity of RLAR was assessed spectrophotometrically by measuring the decrease in NADPH absorbance at $340 \mathrm{~nm}$ for 3 minutes using a spectrophotometer (SECOMAM, Ales Cedex, France). Quercetin was used as the positive controls. The inhibition of RLAR (\%) was calculated using the following equation: $[1-(\triangle A$ sample/min $)-(\triangle A$ blank $/ \mathrm{min}) /(\triangle A$ co ntrol $/ \mathrm{min})-(\triangle A$ blank $/ \mathrm{min})] \times 100 \%$, where $\triangle A$ sample/ $\mathrm{min}$ is the decrease in absorbance over 3 min with reaction solution, test sample, and substrate and $\triangle A \mathrm{control} / \mathrm{min}$ is the same but with DMSO (less than $1 \%$ in total mixture) instead of test sample [23].

2.8. Methylglyoxal-Bovine Serum Albumin Assay Investigating AGE Formation. Bovine serum albumin $(50 \mathrm{mg} / \mathrm{mL})$ was incubated with methylglyoxal $(100 \mathrm{mM})$ in sodium phosphate buffer $(0.1 \mathrm{M}, \mathrm{pH} 7.4)$ in the presence of various concentrations of the compounds (including a control) at $37^{\circ} \mathrm{C}$ for $24 \mathrm{~h}$. Then the fluorescent intensity was measured at an excitation wavelength of $355 \mathrm{~nm}$ and an emission wavelength of $460 \mathrm{~nm}$ with a luminescence spectrometer LS50B (PerkinElmer Ltd., Buckinghamshire, England). The DMSO used as vehicle was found to have no effect on the reaction. All reagents and samples were sterilized by filtration through $0.2 \mathrm{~mm}$ membrane filters [23].

2.9. Evaluation of DPPH Free Radical Scavenging Capacity. $\mathrm{DPPH}$, a stable free radical, was used to determine the free radical-scavenging activity of the extracts. Briefly, a $0.32 \mathrm{mM}$ DPPH solution in $\mathrm{MeOH}$ were prepared, and $180 \mu \mathrm{L}$ of this solution was mixed with $30 \mu \mathrm{L}$ of each sample at concentrations of $0.05-1.0 \mathrm{mg} / \mathrm{mL}$ in DMSO. After $20 \mathrm{~min}$ of incubation in the dark, the decrease in the absorbance of the solution was measured at $570 \mathrm{~nm}$ on a microplate reader (EL800 Universal Microplate reader, Bio-Tek instruments, Winooski, VT, USA). DPPH radical-scavenging activity was expressed as the percentage inhibition (\%) of DPPH in this assay system and was calculated as $(1-\mathrm{B} / \mathrm{A}) \times 100$, where $\mathrm{A}$ and $\mathrm{B}$ are the activities of DPPH without and with the test material, respectively [24].
2.10. Lens Culture and Intracellular Sorbitol Measurement. Lens isolated from 10-week old Sprague-Dawley rats were cultured for $6 \mathrm{~d}$ in TC-199 medium containing $15 \%$ fetal bovine serum, 100 units $/ \mathrm{mL}$ penicillin, and $0.1 \mathrm{mg} / \mathrm{mL}$ streptomycin, under sterile conditions and an atmosphere of 5\% $\mathrm{CO}_{2}$ and $95 \%$ air at $37^{\circ} \mathrm{C}$. Samples were dissolved in DMSO. The lens were divided into three groups (each group $n=3$ ) and cultured in medium containing $30 \mathrm{mM}$ glucose and RLAR-active compounds. Each lens was placed in a well containing $2.0 \mathrm{~mL}$ medium. Sorbitol was identified by HPLC after its derivatization by reaction with benzoic acid to form a fluorescent compound [25].

2.11. Statistical Analysis. Inhibition rates were calculated as percentages (\%) with respect to the control value, and the $\mathrm{IC}_{50}$ value was defined as the concentration at which $50 \%$ inhibition occurred. Data are expressed as mean values \pm standard deviation of triplicate experiments. Data were analyzed using SPSS version 19.0 software. The comparison of mean values was carried out by Student's unpaired $t$-test or one-way analysis of variance (ANOVA), as appropriate; $p<0.05$ was considered statistically significant.

\section{Results}

3.1. Structure Analysis of Isolated Compounds. The MR extract was found to exhibit strong RLAR, AGEs, and antioxidant inhibitory activities, with an $\mathrm{IC}_{50}$ of 4.61, 189.08, and $32.39 \mu \mathrm{g} / \mathrm{mL}$ (Figure 1 and Table 1), respectively. Since this result suggests the likely presence of many AR inhibitors (ARIs) in the extract, attention should be focused on isolating these from this fraction. In order to identify the active compounds from MR, the extract was dissolved in methanol and subjected to repeated chromatography on Sephadex LH-20, silica gel, and reverse-phase C18 columns, to yield compounds 1-10. The structures of isolated compounds were elucidated based on 1-dimensional $\left({ }^{1} \mathrm{H}\right.$ and ${ }^{13} \mathrm{C}$ NMR $)$ and 2-dimensional NMR (HMQC and HMBC) spectral data, by comparing with published spectral data, electronic impact (EI), and fast atom bombardment (FAB) mass spectrometry (MS) data. Isolated compounds were identified as apigenin7-O- $\beta$-D-glucoside (1), luteolin-7-O- $\beta$-D-glucoside (2), penduletin (3), jaceidin (4), apigenin-7-O- $\beta$-D-glucuronide (5), luteolin-7-O- $\beta$-D-glucuronide (6), 3,5-O-di-caffeoylquinic acid (7), 6-hydroxyapigenin (8), apigenin (9), and luteolin (10). The chemical structures of compounds 1-10 isolated from MR are shown in Figure 2 [14-29].

Compound (1). FAB-MS m/z $433[\mathrm{M}+\mathrm{H}]^{+}$. ${ }^{1} \mathrm{H}-\mathrm{NMR}$ $\left(400 \mathrm{MHz}, \mathrm{CD}_{3} \mathrm{OD}, \delta_{\mathrm{H}}\right) \delta 7.87\left(2 \mathrm{H}, \mathrm{d}, J=8.41 \mathrm{~Hz}, \mathrm{H}-2^{\prime} / 6^{\prime}\right)$, $6.91\left(2 \mathrm{H}, \mathrm{d}, J=8.41 \mathrm{~Hz}, \mathrm{H}-3^{\prime} / 5^{\prime}\right), 6.87(1 \mathrm{H}, \mathrm{s}, \mathrm{H}-3), 6.81$ $(1 \mathrm{H}, \mathrm{d}, J=1.73 \mathrm{~Hz}, \mathrm{H}-8), 6.44(1 \mathrm{H}, \mathrm{d}, J=1.73 \mathrm{~Hz}, \mathrm{H}-6)$, $5.15\left(1 \mathrm{H}, \mathrm{d}, J=7.51 \mathrm{~Hz}, \mathrm{H}-1^{\prime \prime}\right), 3.97-3.13\left(6 \mathrm{H}, \mathrm{m}, \mathrm{H}-2^{\prime \prime}, 3^{\prime \prime}\right.$, $4^{\prime \prime}, 5^{\prime \prime}$ and $\left.6 \mathrm{ab}^{\prime \prime}\right),{ }^{13} \mathrm{C}-\mathrm{NMR}\left(100 \mathrm{MHz}, \mathrm{CD}_{3} \mathrm{OD}, \delta_{\mathrm{c}}\right) \delta 180.1$ (C-4), 166.1 (C-7), 162.4 (C-2), 161.8 (C-5), 160.1 (C-4'), $157.1(\mathrm{C}-9), 125.9\left(\mathrm{C}-2^{\prime} / 6^{\prime}\right), 121.7\left(\mathrm{C}-1^{\prime}\right), 116.3\left(\mathrm{C}-3^{\prime} / 5^{\prime}\right)$, 104.8 (C-10), 101.5 (C-3), 98.6 (C-1"), 97.9 (C-6), 94.3 $(\mathrm{C}-8), 77.4\left(\mathrm{C}-3^{\prime \prime}\right), 76.8\left(\mathrm{C}-5^{\prime \prime}\right), 74.0\left(\mathrm{C}-2^{\prime \prime}\right), 69.8\left(\mathrm{C}-4^{\prime \prime}\right)$, $63.8\left(\mathrm{C}-6^{\prime \prime}\right)$. 


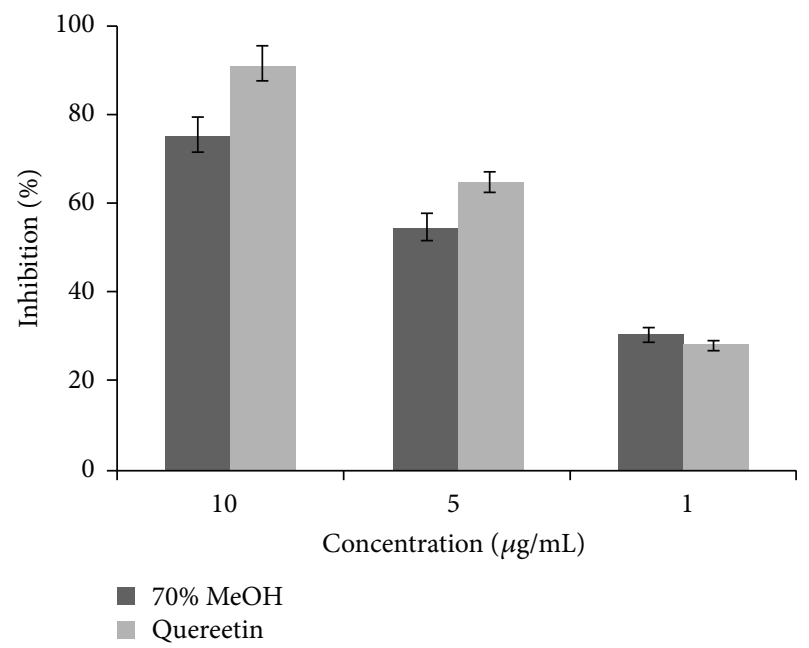

(a)

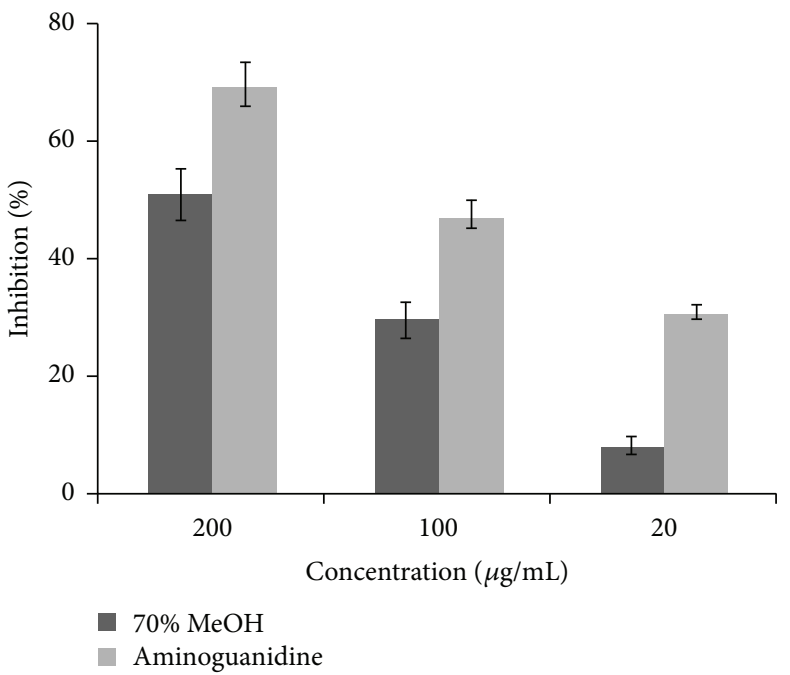

(b)

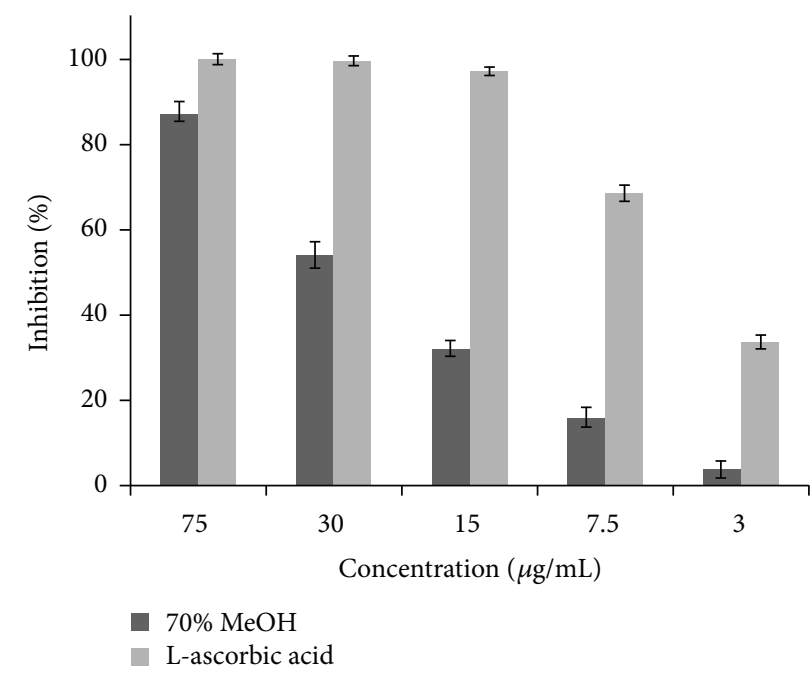

(c)

FIGURE 1: The inhibitory activities by MR crude extract on rat lens aldose reductase (a), advanced glycation end products (b), and DPPH radical-scavenging (c) in various concentrations.

Compound (2). FAB-MS $m / z 449[\mathrm{M}+\mathrm{H}]^{+} .{ }^{1} \mathrm{H}-\mathrm{NMR}$ $\left(400 \mathrm{MHz}, \mathrm{CD}_{3} \mathrm{OD}, \delta_{\mathrm{H}}\right) 7.51(1 \mathrm{H}, \mathrm{dd}, J=8.13,2.07 \mathrm{~Hz}$, H-6 $\left.6^{\prime}\right), 7.44\left(1 \mathrm{H}, \mathrm{d}, J=2.04 \mathrm{~Hz}, \mathrm{H}-2^{\prime}\right), 6.88(1 \mathrm{H}, \mathrm{d}, J=8.12$ $\left.\mathrm{Hz}, \mathrm{H}-5^{\prime}\right), 6.84(1 \mathrm{H}, \mathrm{s}, \mathrm{H}-3), 6.73(1 \mathrm{H}, \mathrm{d}, J=2.10 \mathrm{~Hz}, \mathrm{H}-8)$, $6.49(1 \mathrm{H}, \mathrm{d}, J=2.10 \mathrm{~Hz}, \mathrm{H}-6), 5.11(1 \mathrm{H}, \mathrm{d}, J=7.39 \mathrm{~Hz}$, $\left.\mathrm{H}-1^{\prime \prime}\right)$, 3.85-3.36 (6H, m, H-2", $3^{\prime \prime}, 4^{\prime \prime}, 5^{\prime \prime}$ and $\left.6 \mathrm{ab}^{\prime \prime}\right)$, ${ }^{13} \mathrm{C}-\mathrm{NMR}\left(100 \mathrm{MHz}, \mathrm{CD}_{3} \mathrm{OD}, \delta_{\mathrm{c}}\right) \delta 181.2(\mathrm{C}-4), 164.6$ (C-7), 163.8 (C-2), $161.3(\mathrm{C}-5), 158.2$ (C-9), $153.4\left(\mathrm{C}-4^{\prime}\right)$, $147.8\left(\mathrm{C}-3^{\prime}\right), 125.1\left(\mathrm{C}-6^{\prime}\right), 122.7\left(\mathrm{C}-1^{\prime}\right), 117.0\left(\mathrm{C}-5^{\prime}\right), 115.7$ (C-2'), 106.5 (C-10), 103.2 (C-3), $101.5\left(\mathrm{C}-1^{\prime \prime}\right), 99.7$ (C-6), 96.7 (C-8), $77.1\left(\mathrm{C}-3^{\prime \prime}\right), 76.1\left(\mathrm{C}-5^{\prime \prime}\right), 74.3\left(\mathrm{C}-2^{\prime \prime}\right), 71.1\left(\mathrm{C}-4^{\prime \prime}\right)$, $63.3\left(\mathrm{C}-6^{\prime \prime}\right)$.

Compound (3). FAB-MS $m / z 345[\mathrm{M}+\mathrm{H}]^{+} .{ }^{1} \mathrm{H}-\mathrm{NMR}$ $\left(400 \mathrm{MHz}, \mathrm{CD}_{3} \mathrm{OD}, \delta_{\mathrm{H}}\right) \delta 7.73\left(2 \mathrm{H}, \mathrm{d}, J=8.31 \mathrm{~Hz}, \mathrm{H}-2^{\prime} / 6^{\prime}\right)$, $6.74\left(2 \mathrm{H}, \mathrm{d}, J=8.13 \mathrm{~Hz}, \mathrm{H}-3^{\prime} / 5^{\prime}\right), 6.81(1 \mathrm{H}, \mathrm{s}, \mathrm{H}-8), 3.81$ $\left(9 \mathrm{H}, \mathrm{s},-\mathrm{OCH}_{3}, \mathrm{H}-3 / 6 / 7\right),{ }^{13} \mathrm{C}-\mathrm{NMR}\left(100 \mathrm{MHz}, \mathrm{CD}_{3} \mathrm{OD}, \delta_{\mathrm{c}}\right)$ $\delta 177.9$ (C-4), 159.4 (C-7), 157.3 (C-4'), 156.1 (C-2), 155.7 (C-9), 153.2 (C-5), 138.3 (C-3), 136.9 (C-6), $129.9\left(\mathrm{C}-2^{\prime} / 6^{\prime}\right)$,
$121.7\left(\mathrm{C}-1^{\prime}\right), 116.7\left(\mathrm{C}-3^{\prime} / 5^{\prime}\right), 104.9(\mathrm{C}-10), 96.2(\mathrm{C}-8), 60.1$ $\left(\mathrm{C}-6,-\mathrm{OCH}_{3}\right), 58.7\left(\mathrm{C}-3,-\mathrm{OCH}_{3}\right), 56.3\left(\mathrm{C}-7,-\mathrm{OCH}_{3}\right)$.

Compound (4). FAB-MS $m / z 361[\mathrm{M}+\mathrm{H}]^{+} .{ }^{1} \mathrm{H}-\mathrm{NMR}$ $\left(400 \mathrm{MHz}, \mathrm{CD}_{3} \mathrm{OD}, \delta_{\mathrm{H}}\right) \delta 7.57(1 \mathrm{H}, \mathrm{dd}, J=8.09,1.97 \mathrm{~Hz}$, H-6 $\left.{ }^{\prime}\right), 6.83\left(1 \mathrm{H}, \mathrm{d}, J=8.07 \mathrm{~Hz}, \mathrm{H}-5^{\prime}\right), 6.77(1 \mathrm{H}, \mathrm{d}, J=2.00$ $\left.\mathrm{Hz}, \mathrm{H}-2^{\prime}\right), 6.73(1 \mathrm{H}, \mathrm{s}, \mathrm{H}-8), 3.80\left(9 \mathrm{H}, \mathrm{s},-\mathrm{OCH}_{3}, \mathrm{H}-3 / 4^{\prime} / 6\right)$, ${ }^{13} \mathrm{C}-\mathrm{NMR}\left(100 \mathrm{MHz}, \mathrm{CD}_{3} \mathrm{OD}, \delta_{\mathrm{c}}\right) \delta 178.1(\mathrm{C}-4), 157.8(\mathrm{C}-7)$, 155.3 (C-9), 154.6 (C-2), 151.3 (C-5), 148.9 (C-4'), 147.3 $\left(\mathrm{C}-3^{\prime}\right), 138.5$ (C-3), 130.9 (C-6), $121.7\left(\mathrm{C}-1^{\prime}\right), 120.0\left(\mathrm{C}-6^{\prime}\right)$, $114.7\left(\mathrm{C}-2^{\prime}\right), 111.1\left(\mathrm{C}-5^{\prime}\right), 104.1(\mathrm{C}-10), 95.7(\mathrm{C}-8), 60.6$ $\left(\mathrm{C}-6,-\mathrm{OCH}_{3}\right), 58.6\left(\mathrm{C}-3,-\mathrm{OCH}_{3}\right), 56.9\left(\mathrm{C}-4^{\prime},-\mathrm{OCH}_{3}\right)$.

Compound (5). FAB-MS $m / z 447[\mathrm{M}+\mathrm{H}]^{+}$. ${ }^{1} \mathrm{H}-\mathrm{NMR}$ $\left(400 \mathrm{MHz}, \mathrm{CD}_{3} \mathrm{OD}, \delta_{\mathrm{H}}\right) 7.84\left(2 \mathrm{H}, \mathrm{d}, J=8.77 \mathrm{~Hz}, \mathrm{H}-2^{\prime} / 6^{\prime}\right)$, $6.98\left(2 \mathrm{H}, \mathrm{d}, J=8.73 \mathrm{~Hz}, \mathrm{H}-3^{\prime} / 5^{\prime}\right), 6.84(1 \mathrm{H}, \mathrm{s}, \mathrm{H}-3), 6.76$ $(1 \mathrm{H}, \mathrm{d}, J=1.82 \mathrm{~Hz}, \mathrm{H}-8), 6.49(1 \mathrm{H}, \mathrm{d}, J=1.82 \mathrm{~Hz}, \mathrm{H}-6)$, $5.07\left(1 \mathrm{H}, \mathrm{d}, J=7.07 \mathrm{~Hz}, \mathrm{H}-1^{\prime \prime}\right), 3.96(1 \mathrm{H}, \mathrm{d}, J=9.59 \mathrm{~Hz}$, $\left.\mathrm{H}-5^{\prime \prime}\right), 3.60-3.26\left(3 \mathrm{H}, \mathrm{m}, \mathrm{H}-2^{\prime \prime}, 3^{\prime \prime}\right.$ and $\left.4^{\prime \prime}\right),{ }^{13} \mathrm{C}-\mathrm{NMR}$ $\left(100 \mathrm{MHz}, \quad \mathrm{CD}_{3} \mathrm{OD}, \delta_{\mathrm{c}}\right) \quad \delta \quad 180.1 \quad(\mathrm{C}-4), 171.5 \quad\left(\mathrm{C}-6^{\prime \prime}\right)$, 
TABLE 1: The inhibitory activities of MR crude extract on rat lens aldose reductase (RLAR), DPPH radical scavenging, and advanced glycation end products (AGEs).

\begin{tabular}{lccc}
\hline Entry & \multicolumn{3}{c}{$\mathrm{IC}_{50}(\mu \mathrm{g} / \mathrm{mL})^{1)}$} \\
& RLAR & DPPH & AGEs \\
\hline $70 \% \mathrm{MeOH}$ & $4.61 \pm 0.29^{\mathrm{b}}$ & $32.39 \pm 1.28^{\mathrm{b}}$ & $189.08 \pm 4.19^{\mathrm{b}}$ \\
Quercetin $^{2)}$ & $3.65 \pm 0.10^{\mathrm{a}}$ & - & - \\
L-Ascorbic acid $^{3)}$ & - & $6.60 \pm 0.33^{\mathrm{a}}$ & - \\
Aminoguanidine $^{4)}$ & - & - & $109.10 \pm 3.47^{\mathrm{a}}$ \\
\hline
\end{tabular}

${ }^{1)}$ The $\mathrm{IC}_{50}$ values are defined as mean \pm relative standard derivation (RSD) of half-maximal inhibitory concentrations obtained from three independent experiments performed in duplicate, and the range of the inhibitor concentrations adopted to evaluate $\mathrm{IC}_{50}$ was prepared as follows: (1) RLAR: 1,5 , and $10 \mu \mathrm{g} / \mathrm{mL}$; (2) DPPH: 15,30 , and $75 \mu \mathrm{g} / \mathrm{mL}$; and (3) AGEs: 20,100 , and $200 \mu \mathrm{g} / \mathrm{mL} .{ }^{2)-4)}$ Quercetin, L-ascorbic acid, and aminoguanidine are the positive control for RLAR inhibition, DPPH scavenging, and AGEs inhibition. Values within a column marked with different letters are significantly different from each other $(p<0.05)$.

166.6 (C-7), 163.8 (C-2), 161.7 (C-5), 158.5 (C-4'), 155.7 (C-9), $128.9\left(\mathrm{C}-2^{\prime} / 6^{\prime}\right), 122.1\left(\mathrm{C}-1^{\prime}\right), 118.0\left(\mathrm{C}-3^{\prime} / 5^{\prime}\right), 106.5$ (C-10), 102.8 (C-3), $101.37 \quad\left(\mathrm{C}-1^{\prime \prime}\right), 99.2 \quad(\mathrm{C}-6), 96.3$ (C-8), $78.0\left(\mathrm{C}-3^{\prime \prime}\right), 75.1\left(\mathrm{C}-5^{\prime \prime}\right), 73.9\left(\mathrm{C}-2^{\prime \prime}\right), 71.8\left(\mathrm{C}-4^{\prime \prime}\right)$.

Compound (6). FAB-MS $m / z 463[\mathrm{M}+\mathrm{H}]^{+}$. ${ }^{1} \mathrm{H}-\mathrm{NMR}$ $\left(400 \mathrm{MHz}, \mathrm{CD}_{3} \mathrm{OD}, \delta_{\mathrm{H}}\right) \delta 7.55(1 \mathrm{H}, \mathrm{dd}, J=8.10,2.08 \mathrm{~Hz}$, H-6 $\left.6^{\prime}\right), 7.42\left(1 \mathrm{H}, \mathrm{d}, J=2.07 \mathrm{~Hz}, \mathrm{H}-2^{\prime}\right), 6.94(1 \mathrm{H}, \mathrm{d}, J=8.10$ $\left.\mathrm{Hz}, \mathrm{H}-5^{\prime}\right), 6.82(1 \mathrm{H}, \mathrm{s}, \mathrm{H}-3), 6.79(1 \mathrm{H}, J=2.30 \mathrm{~Hz}, \mathrm{H}-8)$, $6.56(1 \mathrm{H}, \mathrm{d}, J=2.30 \mathrm{~Hz}, \mathrm{H}-6), 5.11(1 \mathrm{H}, \mathrm{d}, J=7.27 \mathrm{~Hz}$, $\left.\mathrm{H}-1^{\prime \prime}\right), 4.08\left(1 \mathrm{H}, \mathrm{d}, J=9.50 \mathrm{~Hz}, \mathrm{H}-5^{\prime \prime}\right), 3.51-3.27(3 \mathrm{H}, \mathrm{m}$, $\mathrm{H}-2^{\prime \prime}, 3^{\prime \prime}$ and $\left.4^{\prime \prime}\right),{ }^{13} \mathrm{C}-\mathrm{NMR}\left(100 \mathrm{MHz}, \mathrm{CD}_{3} \mathrm{OD}, \delta_{\mathrm{c}}\right) \delta$ 180.1 (C-4), 172.1 (C-6"), 165.8 (C-7), 162.8 (C-2), 160.1 (C-5), 152.7 (C-9), 150.7 (C-4'), 146.7 (C-3'), 117.0 (C-6'), $116.82\left(\mathrm{C}-1^{\prime}\right), 114.1\left(\mathrm{C}-5^{\prime}\right), 112.1\left(\mathrm{C}-2^{\prime}\right), 102.9$ (C-10), 100.9 (C-3), 101.1 (C-1"), 98.8 (C-6), 95.1 (C-8), $76.8\left(\mathrm{C}-3^{\prime \prime}\right), 73.4$ $\left(\mathrm{C}-5^{\prime \prime}\right), 74.3\left(\mathrm{C}-2^{\prime \prime}\right), 72.5\left(\mathrm{C}-4^{\prime \prime}\right)$.

Compound (7). FAB-MS $m / z 517[\mathrm{M}+\mathrm{H}]^{+} .{ }^{1} \mathrm{H}-\mathrm{NMR}$ $\left(400 \mathrm{MHz}, \mathrm{CD}_{3} \mathrm{OD}, \delta_{\mathrm{H}}\right) \delta 7.63,7.61(1 \mathrm{H}$ each, $\mathrm{d}, J=16.01$ $\left.\mathrm{Hz}, \mathrm{H}-7 / \mathrm{H}^{\prime} 7^{\prime}\right), 7.12(2 \mathrm{H}$, brs, H-2/H-2'), $6.92(2 \mathrm{H}, \mathrm{dd}$, $\left.J=8.14,2.01 \mathrm{~Hz}, \mathrm{H}-6 / \mathrm{H}-6^{\prime}\right), 6.80(2 \mathrm{H}, \mathrm{dd}, J=7.80,1.22 \mathrm{~Hz}$, $\left.\mathrm{H}-5 / \mathrm{H}-5^{\prime}\right), 6.37,6.29\left(1 \mathrm{H}\right.$ each, $\left.\mathrm{d}, J=16.01 \mathrm{~Hz}, \mathrm{H}-8 / \mathrm{H}-8^{\prime}\right)$, $5.55-5.39(2 \mathrm{H}, \mathrm{m}, \mathrm{H}-3 / \mathrm{H}-5), 4.01(1 \mathrm{H}, \mathrm{dd}, J=9.71,3.26 \mathrm{~Hz}$, $\mathrm{H}-4), 2.39-2.17$ (4H, m, H-2/H-6). ${ }^{13} \mathrm{C}-\mathrm{NMR}(100 \mathrm{MHz}$, $\left.\mathrm{CD}_{3} \mathrm{OD}, \delta_{\mathrm{c}}\right) \delta 176.7(\mathrm{COOH}), 167.9,167.5\left(\mathrm{C}-9 / \mathrm{C}-9^{\prime}\right)$, $148.4,148.2\left(\mathrm{C}-4 / \mathrm{C}-4^{\prime}\right), 146.8,146.6\left(\mathrm{C}-7 / \mathrm{C}^{\prime} 7^{\prime}\right), 145.8$ $\left(\mathrm{C}-3 / \mathrm{C}-3^{\prime}\right), 126.9,126.7\left(\mathrm{C}-1 / \mathrm{C}-1^{\prime}\right), 121.9,121.5\left(\mathrm{C}-6 / \mathrm{C}-6^{\prime}\right)$, 117.8 (C-5/C-5'), 115.7, 115.6 (C-8/C-8'), 115.0 (C-2/C-2'), 73.4 (C-1), 71.9 (C-3), 70.9 (C-5), 69.8 (C-4), 37.9 (C-6), $36.7(\mathrm{C}-2)$.

Compound (8). FAB-MS $m / z 287[\mathrm{M}+\mathrm{H}]^{+} .{ }^{1} \mathrm{H}-\mathrm{NMR}$ $\left(400 \mathrm{MHz}, \quad \mathrm{CD}_{3} \mathrm{OD}, \quad \delta_{\mathrm{H}}\right) \delta 7.74(2 \mathrm{H}, \mathrm{d}, J=8.21 \mathrm{~Hz}$, $\left.\mathrm{H}-2^{\prime} / \mathrm{H}-6^{\prime}\right), 6.84\left(2 \mathrm{H}, \mathrm{d}, J=8.14 \mathrm{~Hz}, \mathrm{H}-3^{\prime} / \mathrm{H}-5^{\prime}\right), \quad 6.76$ $(1 \mathrm{H}, \mathrm{s}, \mathrm{H}-3), 6.40(1 \mathrm{H}, \mathrm{s}, \mathrm{H}-8) ;{ }^{13} \mathrm{C}-\mathrm{NMR}(100 \mathrm{MHz}$, $\left.\mathrm{CD}_{3} \mathrm{OD}, \delta_{c}\right) \delta 181.6(\mathrm{C}-4), 164.3(\mathrm{C}-2), 159.7$ (C-7), $158.6\left(\mathrm{C}-4^{\prime}\right), 157.3$ (C-5), 153.1 (C-9), 145.9 (C-6), 129.0 (C-2'/C-6'), $120.4\left(\mathrm{C}-1^{\prime}\right), 115.6\left(\mathrm{C}-3^{\prime} / \mathrm{C}-5^{\prime}\right), 106.1$ (C-10), 102.3 (C-3), 94.6 (C-8).

Compound (9). EI-MS $m / z 271[\mathrm{M}+\mathrm{H}]^{+} .{ }^{1} \mathrm{H}-\mathrm{NMR}$ $\left(400 \mathrm{MHz}, \quad \mathrm{CD}_{3} \mathrm{OD}, \quad \delta_{\mathrm{H}}\right) \delta 7.82(2 \mathrm{H}, \mathrm{d}, J=8.15 \mathrm{~Hz}$,
H-2 $\left.{ }^{\prime} / \mathrm{H}-6^{\prime}\right), \quad 6.97 \quad\left(2 \mathrm{H}, \quad \mathrm{d}, \quad J=8.15 \mathrm{~Hz}, \quad \mathrm{H}-3^{\prime} / \mathrm{H}-5^{\prime}\right), \quad 6.79$ $(1 \mathrm{H}, \quad \mathrm{s}, \quad \mathrm{H}-3), \quad 6.58(1 \mathrm{H}, \quad \mathrm{d}, \quad J=2.11 \mathrm{~Hz}, \mathrm{H}-8), \quad 6.39$ $(1 \mathrm{H}, J=2.11 \mathrm{~Hz}, \mathrm{H}-6) ;{ }^{13} \mathrm{C}-\mathrm{NMR}\left(100 \mathrm{MHz}, \mathrm{CD}_{3} \mathrm{OD}, \delta_{\mathrm{c}}\right)$ $\delta 180.0$ (C-4), 165.7 (C-2), 163.9 (C-7), 160.9 (C-5), $160.2\left(\mathrm{C}-4^{\prime}\right), 158.6(\mathrm{C}-9), 129.1\left(\mathrm{C}-2^{\prime} / \mathrm{C}-6^{\prime}\right), 120.9\left(\mathrm{C}-1^{\prime}\right)$, $115.7\left(\mathrm{C}-3^{\prime} / \mathrm{C}-5^{\prime}\right), 101.3$ (C-10), 100.1 (C-3), 98.8 (C-6), 94.0 (C-8).

Compound (10). EI-MS $m / z 287[\mathrm{M}+\mathrm{H}]^{+} .{ }^{1} \mathrm{H}-\mathrm{NMR}$ $\left(400 \mathrm{MHz}, \mathrm{CD}_{3} \mathrm{OD}, \delta_{\mathrm{H}}\right) \delta 7.51(1 \mathrm{H}, \mathrm{dd}, J=9.17,2.02 \mathrm{~Hz}$,

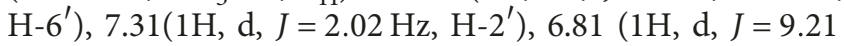
$\left.\mathrm{Hz}, \mathrm{H}-5^{\prime}\right), 6.68(1 \mathrm{H}, \mathrm{s}, \mathrm{H}-3), 6.47(1 \mathrm{H}, \mathrm{d}, J=2.03 \mathrm{~Hz}, \mathrm{H}-8)$, $6.18(1 \mathrm{H}, \quad \mathrm{d}, \quad J=2.03 \mathrm{~Hz}, \mathrm{H}-6) .{ }^{13} \mathrm{C}-\mathrm{NMR} \quad(100 \mathrm{MHz}$, $\left.\mathrm{CD}_{3} \mathrm{OD}, \delta_{c}\right) \delta 180.6(\mathrm{C}-4), 163.7(\mathrm{C}-2), 162.1 \quad(\mathrm{C}-7)$, 160.7 (C-5), 158.5 (C-9), $148.2\left(\mathrm{C}-4^{\prime}\right), 144.9\left(\mathrm{C}-3^{\prime}\right), 120.5$ $\left(\mathrm{C}-6^{\prime}\right), 118.7\left(\mathrm{C}-1^{\prime}\right), 116.2\left(\mathrm{C}-5^{\prime}\right), 113.8\left(\mathrm{C}-2^{\prime}\right), 104.2(\mathrm{C}-10)$, 102.7 (C-3), 99.2 (C-6), 96.8 (C-8).

3.2. AR Inhibitory Activities of the Isolated Compounds. The inhibitory activities of compounds 1-10 on RLAR were evaluated. As shown in Table 2, compounds 6 and 7 showed the strongest inhibition against RLAR $\left(\mathrm{IC}_{50}=0.85\right.$ and $0.72 \mu \mathrm{M}$, resp.). In addition, compounds $2\left(\mathrm{IC}_{50}=1.12 \mu \mathrm{M}\right)$, $5\left(\mathrm{IC}_{50}=1.16 \mu \mathrm{M}\right), 9\left(\mathrm{IC}_{50}=1.71 \mu \mathrm{M}\right)$, and $10\left(\mathrm{IC}_{50}=1.42\right.$ $\mu \mathrm{M})$ were found to possess significant RLAR inhibitory activity in vitro $(2>5>10>9)$, compared to quercetin $\left(\mathrm{IC}_{50}=1.21 \mu \mathrm{M}\right)$, a well-known ARI. Compounds 3, 4 , and 8 were inactive.

3.3. Inhibitory Activities of AGEs. The methylglyoxal-BSA assay was used specifically to investigate inhibitors of protein glycation formation in $\mathrm{MR}$ extract and was performed according to the method characterized by Li et al. [30]. The MR extract showed high AGE inhibitory activity with an $\mathrm{IC}_{50}$ value of $189.08 \mu \mathrm{g} / \mathrm{mL}$. In addition, we compared inhibition of the formation of advanced glycation by compounds 1-10 with that achieved by AG, a well-known AGE inhibitor. As shown in Table 2, compounds 6 and 10 (luteolin-7-O- $\beta$ D-glucuronide and luteolin) had $\mathrm{IC}_{50}$ values of 3.39 and $6.01 \mu \mathrm{M}$, respectively, and were found to be more effective than AG in inhibiting the formation of advanced glycation, while other compounds were inactive and showed varied low inhibitory effects ranging from $9.06-16.25 \%$ at a concentration of $20 \mu \mathrm{g} / \mathrm{mL}$. Our results showed that compounds 6 and 10 , which contain a glucuronide at position 7 in the $\mathrm{A}$ ring and di-hydroxyl groups in the $\mathrm{B}$ ring of the flavonol skeleton, exhibited the highest AGE inhibitory activity.

3.4. Antioxidant Activities of the Isolated Compounds. The MR extract exhibited potent inhibition on DPPH free radical-scavenging activity $(32.39 \mu \mathrm{g} / \mathrm{mL})$ compared to the positive control L-ascorbic, which had an $\mathrm{IC}_{50}$ value of $6.60 \mu \mathrm{g} / \mathrm{mL}$. The scavenging activities of the ten compounds isolated from MR were evaluated using the same method (Table 2). Of the tested compounds, compounds 2 and 6 had the highest $\mathrm{IC}_{50}$ values: 7.24 and $8.92 \mu \mathrm{M}$, respectively. Compounds 5, 7, 9, and 10 also showed strong scavenging activity with $\mathrm{IC}_{50}$ values of $10.58-15.63 \mu \mathrm{M}$, compared to the positive control, $\mathrm{L}$-ascorbic acid $\left(\mathrm{IC}_{50}=3.75 \mu \mathrm{M}\right)$. 
<smiles>[R]c1ccc(-c2oc3cc([R])c([R])c(O)c3c(=O)c2[R])cc1[R]</smiles>

Flavonoid derivatives<smiles>O=C(O)C1(O)C[C@H](O)[C@H](O)[C@H](O)[C@H]1O</smiles>

Quinic acid<smiles>CC(=O)/C=C/c1ccc(O)c(O)c1</smiles>

Caffeoyl

3,5-O-di-caffeoylquinic acid (7) : $\mathrm{R}_{6}=$ Caffeoyl; $\mathrm{R}_{7}=$ Caffeoyl

\begin{tabular}{|c|c|c|c|c|c|}
\hline Flavonoid derivatives & $\mathrm{R}_{1}$ & $\mathrm{R}_{2}$ & $\mathrm{R}_{3}$ & $\mathrm{R}_{4}$ & $\mathrm{R}_{5}$ \\
\hline Agipenin-7-O- $\beta$-D-glucuronide (1) & -O- $\beta$-D-glucopyranose & $\mathrm{H}$ & $\mathrm{H}$ & $\mathrm{H}$ & $\mathrm{OH}$ \\
\hline Luteolin-7-O- $\beta$-D-glucuronide (2) & -O- $\beta$-D-glucopyranose & $\mathrm{H}$ & $\mathrm{H}$ & $\mathrm{OH}$ & $\mathrm{OH}$ \\
\hline Penduletin (3) & $-\mathrm{OCH}_{3}$ & $-\mathrm{OCH}_{3}$ & $-\mathrm{OCH}_{3}$ & $\mathrm{H}$ & $\mathrm{OH}$ \\
\hline Jaceidin (3) & $\mathrm{OH}$ & $-\mathrm{OCH}_{3}$ & $-\mathrm{OCH}_{3}$ & $\mathrm{OH}$ & $-\mathrm{OCH}_{3}$ \\
\hline Agipenin-7-O- $\beta$-D-glucunide (5) & -O- $\beta$-D-glucopyranosiduronic acid & $\mathrm{H}$ & $\mathrm{H}$ & $\mathrm{H}$ & $\mathrm{OH}$ \\
\hline Luteolin-7-O- $\beta$-D-glucunide (6) & -O- $\beta$-D-glucopyranosiduronic acid & $\mathrm{H}$ & $\mathrm{H}$ & $\mathrm{OH}$ & $\mathrm{OH}$ \\
\hline 6-Hydroxyapigenin (8) & $\mathrm{OH}$ & $\mathrm{OH}$ & $\mathrm{H}$ & $\mathrm{H}$ & $\mathrm{OH}$ \\
\hline Apigenin (9) & $\mathrm{OH}$ & $\mathrm{H}$ & $\mathrm{H}$ & $\mathrm{H}$ & $\mathrm{OH}$ \\
\hline Luteolin (10) & $\mathrm{OH}$ & $\mathrm{H}$ & $\mathrm{H}$ & $\mathrm{OH}$ & $\mathrm{OH}$ \\
\hline
\end{tabular}

FIgURE 2: The chemical structures of compounds isolated from Matricaria recutita L.

Compounds 3, 4, and 8 had almost no effect on DPPH free radical scavenging activity.

3.5. Inhibitory Activities of Active Compounds on Sorbitol Accumulation. We also investigated the effects of RLAR inhibitory compounds on sorbitol accumulation in isolated rat lens (results shown in Table 3). Compounds 1, 2, 5, 6, 7, 9 , and 10 effectively inhibited sorbitol accumulation by $51.02,95.23,80.27,91.83,86.39,87.07$, and $91.83 \%$ at concentration of $5 \mu \mathrm{g} / \mathrm{mL}$, respectively. The positive control (quercetin) inhibited sorbitol accumulation in rat lens by $85.71 \%$ and reduced sorbitol levels in culture medium containing a high glucose concentration.

3.6. Interaction Analysis of Active Compounds Isolated with $A R$. To explore the binding of flavonoids and AR, molecular docking studies were carried. Docking interactions showed that the flavonoids isolated from MR bind stably with AR (Figure 3). Compounds bind to the active site of $\mathrm{AR}$ at Ser-302; His-110; Ala-299; Leu-301 and 302, and Trp-20, 48 , and 111 residues. All seven compounds occupied the active site and interacted with the surrounding residues at different orientations. The molecular docking method can reveal the nature of ligand binding at active site for various compounds. Our molecular docking simulation suggested that the strategy for screening AR inhibitor from natural products is reliable and can be used to distinguish the specific inhibitors from false positives.

\section{Discussion}

The flavonoids and derivatives are an interesting group of natural products that are found in various widely distributed plants, and most of these compounds are isolated from medicinal plants $[31,32]$. Previous investigations into the inhibitory activities of flavonoids and their derivatives reported that luteolin (10) and luteolin-5-O- $\beta$-D-glucopyranoside isolated from Cirsium maackii (A perennial thistle of Asteraceae family) showed inhibitory effects on RLAR comparable to those of the positive control (quercetin). This study indicated that the inhibitory activity of luteolin-5-O$\beta$-D-glucopyranoside on RLAR was almost 1.58 times 
TABLE 2: Inhibitory activities of compounds isolated from Matricaria recutita L. on rat lens aldose reductase (RLAR), DPPH radical scavenging, and advanced glycation end products (AGEs).

\begin{tabular}{|c|c|c|c|}
\hline \multirow{2}{*}{ Compounds } & \multicolumn{3}{|c|}{$\mathrm{IC}_{50}(\mu \mathrm{M})^{1)}$} \\
\hline & RLAR & $\mathrm{DPPH}$ & AGEs \\
\hline Agipenin-7-O- $\beta$-D-glucoside (1) & $4.25 \pm 0.07^{\mathrm{d}}$ & $>25.0$ & NI \\
\hline Luteolin-7-O- $\beta$-D-glucoside (2) & $1.12 \pm 0.02^{\mathrm{b}}$ & $7.24 \pm 0.38^{\mathrm{b}}$ & NI \\
\hline Penduletin (3) & $\mathrm{NI}^{2)}$ & $\mathrm{NI}$ & $\mathrm{NI}$ \\
\hline Jaceidin (4) & NI & NI & NI \\
\hline Agipenin-7-O- $\beta$-D-glucuronide (5) & $1.16 \pm 0.04^{\mathrm{b}}$ & $10.58 \pm 0.47^{\mathrm{bc}}$ & NI \\
\hline Luteolin-7-O- $\beta$-D-glucuronide (6) & $0.85 \pm 0.02^{\mathrm{a}}$ & $8.92 \pm 0.21^{\mathrm{b}}$ & $3.39 \pm 0.17^{\mathrm{a}}$ \\
\hline 3,5-O-di-caffeoylquinic acid (7) & $0.72 \pm 0.02^{\mathrm{a}}$ & $12.34 \pm 0.63^{\mathrm{bc}}$ & NI \\
\hline 6-Hydroxyapigenin (8) & $\mathrm{NI}$ & $\mathrm{NI}$ & NI \\
\hline Apigenin (9) & $1.72 \pm 0.04^{\mathrm{c}}$ & $15.63 \pm 0.34^{\mathrm{c}}$ & NI \\
\hline Luteolin (10) & $1.42 \pm 0.03^{\mathrm{bc}}$ & $11.53 \pm 0.38^{\mathrm{bc}}$ & $6.01 \pm 0.38^{\mathrm{b}}$ \\
\hline Quercetin $^{3)}$ & $1.21 \pm 0.04^{\mathrm{b}}$ & - & - \\
\hline L-Ascorbic acid ${ }^{4)}$ & - & $3.75 \pm 0.17^{\mathrm{a}}$ & - \\
\hline Aminoguanidine $e^{5)}$ & - & - & $98.69 \pm 5.31^{c}$ \\
\hline
\end{tabular}

TABLE 3: Inhibitory effects of rat lens aldose reductase-active compounds of Matricaria recutita L. on sorbitol accumulation in rat lens.

\begin{tabular}{lcc}
\hline Compounds & $\begin{array}{c}\text { Sorbitol content } \\
(\mathrm{mg}) / \text { lens wet } \\
\text { weight }(\mathrm{g})\end{array}$ & $\begin{array}{c}\text { Inhibition } \\
(\%)\end{array}$ \\
\hline Sorbitol free by G free & $\begin{array}{c}\text { No detection } \\
1.47 \pm 0.02\end{array}$ & - \\
Control by G & $0.21 \pm 0.01^{\mathrm{a}}$ & $85.71 \pm 3.23^{\mathrm{a}}$ \\
Quercetin ${ }^{1)}$ by G+ quercetin & $0.72 \pm 0.01^{\mathrm{c}}$ & $51.02 \pm 1.68^{\mathrm{c}}$ \\
Agipenin-7-O- $\beta$-D-glucoside (1) & $0.07 \pm 0.01^{\mathrm{a}}$ & $95.23 \pm 8.18^{\mathrm{a}}$ \\
Luteolin-7-O- $\beta$-D-glucoside (2) & & \\
Agipenin-7-O- $\beta$-D-glucuronide & $0.29 \pm 0.01^{\mathrm{b}}$ & $80.27 \pm 2.78^{\mathrm{b}}$ \\
(5) & & \\
Luteolin-7-O- $\beta$-D-glucuronide & $0.12 \pm 0.01^{\mathrm{a}}$ & $91.83 \pm 6.74^{\mathrm{a}}$ \\
(6) & $0.20 \pm 0.01^{\mathrm{a}}$ & $86.39 \pm 4.28^{\mathrm{a}}$ \\
3,5-O-di-caffeoylquinic acid (7) & $0.19 \pm 0.01^{\mathrm{a}}$ & $87.07 \pm 4.48^{\mathrm{a}}$ \\
Apigenin (9) & $0.12 \pm 0.01^{\mathrm{a}}$ & $91.83 \pm 6.87^{\mathrm{a}}$ \\
Luteolin (10) &
\end{tabular}

${ }^{1)}$ Quercetin was used as the positive control. Results are presented as mean \pm SD $(n=3)$. Values within a column marked with different letters are significantly different from each other $(p<0.05)$. Samples concentration was used at $5 \mu \mathrm{g} / \mathrm{mL}$ on sorbitol accumulation in rat lens.

greater than that of its precursor [33]. In our results, luteolin7-O- $\beta$-D-glucoside (2) (monoglycosylation) showed to be higher than luteolin (10) (aglycone) on RLAR. Monoglycosylation and diglycosylation at luteolin (10) elevated its inhibitory potency significantly, suggesting that the RLAR inhibitory activity of luteolin (10) is strongly related to the number and position of sugar moieties. Luteolin-7-O- $\beta$-Dglucoside (2) and luteolin-7-O-rutinoside were isolated from
Colocasia esculenta by Li et al. [34], and a number of sugar moieties at the same positions in the flavonoid skeleton were shown to have different inhibitory activities on RLAR [34]. However, the effects of these products on sorbitol accumulation were not reported. Jung et al. (2011) suggested that the addition of a sugar group to the flavonoid skeleton in position 3 (quercetin-3-O-glucoside and quercetin-3-O- $\beta$-Dgalactoside) may be responsible for a loss of RLAR inhibitory activity compared to its precursor (quercetin) [35]. In addition, quercetin derivatives (quercetin-3-O-D-glucoside, quercetin-3-O- $\beta$-D-glucuronide, quercetin-3-O- $\beta$-D-galactoside, and quercetin-3-O- $\beta$-D-rutinoside) isolated from the extracts of Nelumbo nucifera leaves exhibited the most potent inhibitory activity in RLAR, AGEs, and oxidative stress [35]. This work indicates that sugar moieties in flavonoid skeletons may be implicated in the potency of RLAR inhibitory effects. The RLAR inhibitory activities of flavonoid compounds $1,2,6,7,9$, and 10 isolated from plant sources were as follows: compound $1(23.0 \mu \mathrm{M})$, compound $2(0.99 \mu \mathrm{M})$, compound $6(3.1 \mu \mathrm{M})$, compound 7 $(0.19 \mu \mathrm{M})$, compound $9(2.2 \mu \mathrm{M})$, and compound 10 $(0.45 \mu \mathrm{M})$ [36-39]. These reported data were similar to the SAR data of our flavonoids on RLAR.

Compounds 1 and 2 are glucosides, 3 and 4 are methoxy aglycones, compounds 5 and 6 are glucuronides, and compounds 8-10 are aglycones. Flavonoid glucuronides showed higher inhibitory activities against AR than their precursor and glycosides. Furthermore, di-hydroxy B-ring flavonoids (compounds 2, 6, and 10) showed higher inhibitory activities against AR than mono-hydroxy B-ring flavonoids (compounds 1, 5, and 9). On the other hand, methoxy (compounds 3 and 4) and tri-hydroxy A-ring flavonoids (compound 8) did not show inhibitory activities against RLAR. In addition, Matsuda et al. [39] reported that the RLAR inhibitory effects 


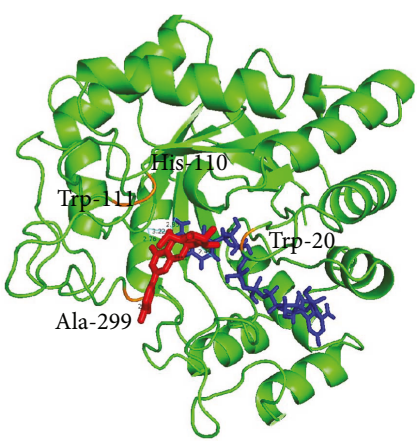

(a)

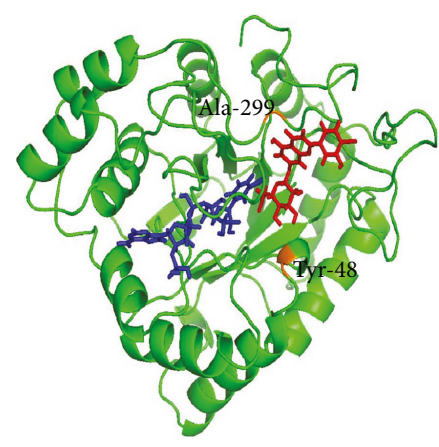

(b)

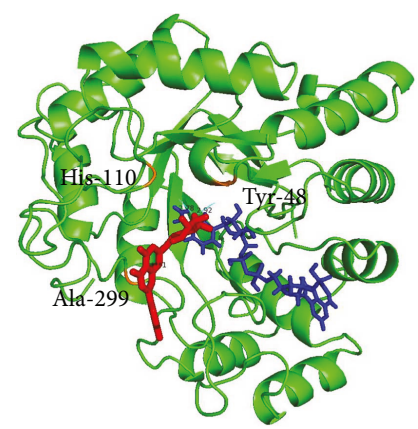

(c)

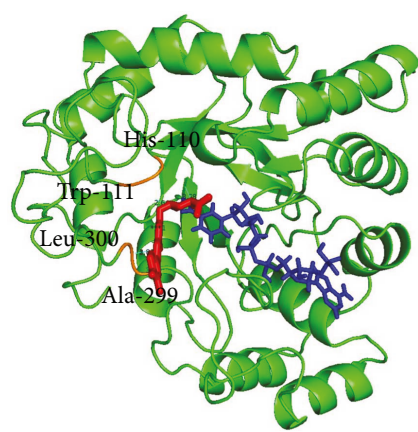

(d)

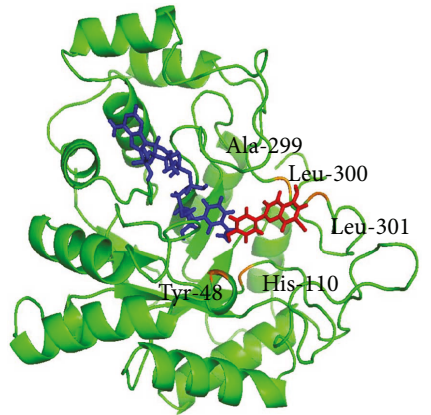

(f)

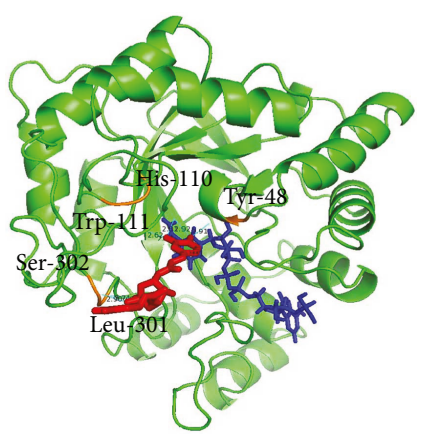

(e)

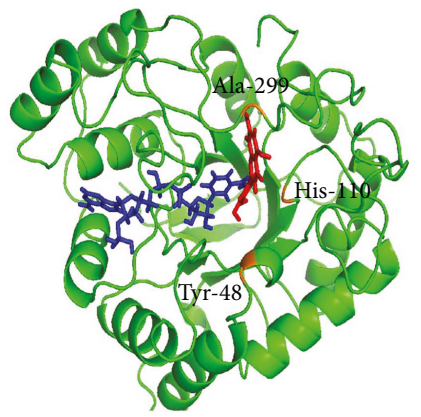

(g)

Figure 3: Docking models of apigenin-7-O- $\beta$-D-glucoside (a), luteolin-7-O- $\beta$-D-glucoside (b), apigenin-7-O- $\beta$-D-glucuronide (c), luteolin7-O- $\beta$-D-glucuronide (d), 3,5-O-di-caffeoylquinic acid (e), apigenin (f), and luteolin (g). The structure of aldose reductase is in green; the structures of the ligands are in red; the interactions of the residues with the ligands are shown in orange.

of flavonoid derivatives depend on the number and site of hydroxyl, methoxyl, and sugar moieties in the aromatic ring of the flavonoid skeleton [39]. On the other hand, other compounds (excluding 3,4, and 8) showed similar activities on DPPH radical-scavenging activities. Based on these results, a significant relationship between RLAR inhibitory activities and protective properties against oxidative stress was observed in flavonoids.

According to the many structural properties of flavonoids, the inhibition of AGE formation has been reported [40]. Compound 6 showed stronger antiglycation effect than both compounds 10 (luteolin) and 2. Increasing the number of glucuronides at position 7 of the A ring (compound 6) in the skeleton of compound 10 increased its inhibitory activities on protein glycation. Jung et al. [41] previously reported that the number of hydroxyl groups at positions 3 and 4 of the $\mathrm{B}$ ring in compound 10 increased its inhibitory activities against each stage of protein glycation. Compounds 3, 4, and 8 did not show inhibitory activities on the formation of advanced glycation. For this reason, our SAR data suggest that a hydroxyl group at position 3 of the $\mathrm{B}$ ring and a glucuronide group at position 7 of the A ring may contribute to the AGE inhibitory activity. The flavonoids isolated from MR do have an established SAR to explain the antiglycation activity demonstrated in the assays above. Based on these results, flavonoids showed a significant relationship between AGE inhibitory activities and functional groups in the flavonoid skeleton. Compounds 1, 2, 5, 6, 7, 9, and 10 showed different RLAR inhibitory activities (compound $7>6>2>5>10>9>1$ ). On the other hand, the strong inhibition of sorbitol accumulation was observed, in the following order: compound $2(95.23 \%)>6$ and $10(91.83 \%)>9$ $(87.07 \%)>7(86.39 \%)>5(80.27 \%)>1(51.02 \%)$. Previously, Kim et al. [42], Lee et al. [43], and Kim et al. [44] reported that 


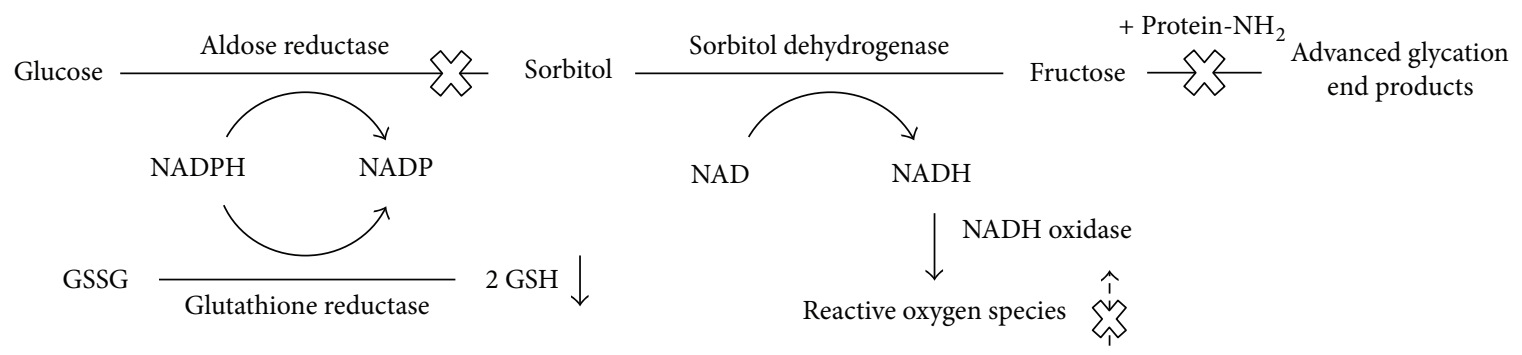

Wnhibition points of Matricaria recutita L. and its constituents
i
$i \downarrow$ Points of increasing or decreasing

FIGURE 4: Inhibition points of Matricaria recutita L. and its constituents on polyol pathway. GSH: glutathione; GSSG: glutathione disulfide; NAD: nicotinamide adenine dinucleotide; NADH: oxidoreductase-induced nicotinamide adenine dinucleotide; NADP: nicotinamide adenine dinucleotide phosphate; NADPH: oxidoreductase-induced nicotinamide adenine dinucleotide phosphate.

the flavonoid derivatives isolated from extracts of Paulownia coreana, Quercus acutissima, and Chamaecyparis obtusa exerted inhibitory effects on sorbitol accumulation based on the RLAR assay.

In this study, different inhibitory activities were seen in vitro and ex vivo and were related to the structures of the flavonoids. Therefore, this result suggests that AR inhibition and sorbitol accumulation may be affected by the structures of the flavonoids. However, the mechanism of MR and its constituents on inhibitory effects of AR and AGEs formation have not yet been found. Therefore, more physiological studies of the MR will be needed for the development of phytomedicine and functional food source.

In the present study, the RLAR, AGE, and DPPH radicalscavenging inhibitory activities of MR and its constituents were investigated. MR and its constituents showed high inhibitory activities in three in vitro assays (Figure 4), and their considerable beneficial effects on diabetic complications would make MR a good ingredient in functional foods. Furthermore, the flavonoids (compounds 1, 2, 5, 6, 9, and 10) and polyphenol (compound 7) isolated from MR showed potent inhibitory activities on sorbitol accumulation in isolated rat lens. This research may provide fundamental knowledge for the development of RLAR, AGE, and antioxidant inhibitors from MR and/or its components. These results suggest that $\mathrm{MR}$ and its constituents can be potent functional food ingredients as RLAR and AGE inhibitors and can be used as naturotherapy for diabetic complications, including oxidative stress.

\section{Conflicts of Interest}

The authors declare that there are no potential competing interests relevant to this paper.

\section{Acknowledgments}

This research was supported by Hallym University Research Fund 2017 (H20170035).

\section{References}

[1] S. M. Bandeira, L. J. S. Fonseca, G. S. Guedes, L. A. Rabelo, M. O. F. Goulart, and S. M. L. Vasconcelos, "Oxidative stress as an underlying contributor in the development of chronic complications in diabetes mellitus," International Journal of Molecular Sciences, vol. 14, no. 2, pp. 3265-3284, 2013.

[2] S. V. Bhadada, V. K. Vyas, and R. K. Goyal, "Protective effect of Tephrosia purpurea in diabetic cataract through aldose reductase inhibitory activity," Biomedicine \& Pharmacotherapy, vol. 83, pp. 221-228, 2016.

[3] S. B. Kim, S. B. Hwang, S. H. Suh, H. W, and S. S. Lim, "Phytochemical analysis of Agrimonia pilosa ledeb, its antioxidant activity and aldose reductase inhibitory potential," International Journal of Molecular Sciences, vol. 18, no. 2, p. 379, 2017.

[4] R. Rahimi, S. Nikfar, B. Larijani, and M. Abdollahi, "A review on the role of antioxidants in the management of diabetes and Its complications," Biomedicine \& Pharmacotherapy, vol. 59, no. 7, pp. 365-373, 2005.

[5] C. S. Kim, J. H. Kim, Y. M. Lee, E. J. Sohn, and J. S. Kim, "Esculetin, a coumarin derivative, inhibits aldose reductase activity in vitro and cataractogenesis in galactose-fed rats," Biomolecules \& Therapeutics, vol. 24, no. 2, pp. 178-183, 2016.

[6] S. H. Hwang, Z. Q. Wang, and S. S. Lim, "Chemo-enzymatic synthesis of vinyl and L-ascorbyl phenolates and their inhibitory effects on advanced glycation end products," Food Chemistry, vol. 214, pp. 726-735, 2017.

[7] K. Nowotny, T. Jung, A. Hohn, D. Weber, D. Weber, and T. Grune, "Advanced glycation end products and oxidative stress in type 2 diabetes mellitus," Biomolecules, vol. 5, no. 1, pp. 194-222, 2015.

[8] Q. Wei, X. Ren, Y. Jiang, H. Jin, N. Liu, and J. Li, “Advanced glycation end products accelerate rat vascular calcification through RAGE/oxidative stress," BMC Cardiovascular Disorders, vol. 13, no. 1, 2013.

[9] M. K. Kang, S. H. Park, Y. J. Choi, D. K. Shin, and Y. H. Kang, "Chrysin inhibits diabetic renal tubulointerstitial fibrosis through blocking epithelial to mesenchymal transition," Journal of Molecular Medicine, vol. 93, no. 7, pp. 759772,2015

[10] T. H. Kim, J. K. Kim, Y. H. Kang, J. Y. Lee, I. J. Kang, and S. S. Lim, "Aldose reductase inhibitory activity of compounds 
from Zea mays L," Biomed Research International, vol. 2013, Article ID 727143, 8 pages, 2013.

[11] Y. S. Oh, "Bioactive compounds and their neuroprotective effects in diabetic complications," Nutrients, vol. 8, no. 8, p. $472,2013$.

[12] D. H. Lee and C. S. Lee, "Flavonoid myricetin inhibits TNF$\alpha$-stimulated production of inflammatory mediators by suppressing the Akt, mTOR and NF- $\kappa$ B pathways in human keratinocytes," European Journal of Pharmacology, vol. 784, pp. 164-172, 2016.

[13] N. Shivanna, M. Naika, F. Khanum, and V. L. Kaul, “Antioxidant, anti-diabetic and renal protective properties of Stevia rebaudiana," Journal of Diabetes and its Complications, vol. 27, no. 2, pp. 103-113, 2013.

[14] H. A. Jung, M. D. N. Islam, Y. S. Kwon et al., "Extraction and identification of three major aldose reductase inhibitors from Artemisia montana," Food and Chemical Toxicology, vol. 49, no. 2, pp. 376-384, 2011.

[15] H. Sebal, M. A. Jabri, A. Souli et al., "Antidiarrheal and antioxidant activities of chamomile (Matricaria recutita L.) decoction extract in rats," Journal of Ethnopharmacology, vol. 152, no. 2, pp. 327-332, 2014.

[16] F. H. Al-Hashem, "Gastroprotective effects of aqueous extract of Chamomilla recutita against ethanol-induced gastric ulcers," Saudi Medical Journal, vol. 31, no. 11, pp. 1211-1216, 2010.

[17] Z. Matić, Z. Juranić, K. Savikin, G. Zdunić, N. Nađvinski, and D. Gođevac, "Chamomile and marigold tea: chemical characterization and evaluation of anticancer activity," Phytotherapy Research, vol. 27, no. 6, pp. 852-858, 2013.

[18] A. Viapiana, W. Struck-Lewicka, P. Konieczynski, M. Wesolowski, and R. Kaliszan, "An approach based on HPLC-fingerprint and chemometrics to quality consistency evaluation of Matricaria chamomilla L. commercial samples," Frontiers in Plant Science, vol. 7, 2016.

[19] J. K. Srivastava and S. Gupta, "Extraction, characterization, stability and biological activity of flavonoids isolated from chamomile flowers," Molecular and Cellular Pharmacology, vol. 1, no. 3, pp. 138-147, 2009.

[20] M. A. Jabri, S. Mohsen, M. Lamjed, and S. Hichem, "Chamomile (Matricaria recutita L.) decoction extract inhibits in vitro intestinal glucose absorption and attenuates high fat diet-induced lipotoxicity and oxidative stress," Biomedicine \& Pharmacotherapy, vol. 87, pp. 153-159, 2017.

[21] D. N. Olennikov and N. I. Kashchenko, "New acylated apigenin glycosides from edge flowers of Matricaria chamomilla," Chemistry of Natural Compounds, vol. 52, no. 6, pp. 996-999, 2016.

[22] M. H. Li, J. K. Kim, J. M. Jang, C. B. Cui, and S. S. Lim, "Analysis of the inhibitory activity of Abeliophyllum distichum leaf constituents against aldose reductase by using high-speed counter current chromatography," Archives of Pharmacal Research, vol. 36, no. 9, pp. 1104-1112, 2013.

[23] J. H. Paek, K. H. Shin, Y. H. Kang, J. K. Lee, and S. S. Lim, "Rapid identification of aldose reductase inhibitory compounds from Perilla frutescens," BioMed Research International, vol. 2013, Article ID 679463, 8 pages, 2013.

[24] X. F. Yin, Y. E. Jeon, H. C. Chung, S. Y. Choung, J. H. Shim, and I. K. Kang, "In vitro efficacy evaluation for prevention of diabetes and diabetic complications using Aster sphathulifolius," Food Science and Biotechnology, vol. 24, no. 1, pp. 301-306, 2015.
[25] Y. S, Lee, S. H. Kim, J. K. Jung, C. H. P. Kim, and S. S. Lim, "Aldose reductase inhibitory compounds from Glycyrrhiza uralensis," Biological and Pharmaceutical Bulletin, vol. 33, no. 5, pp. 917-921, 2010.

[26] X. Li, Y. Lu, R. Deng, T. Zheng, and L. Lv, "Chemical components from the haulm of Artemisia selengensis and the inhibitory effect on glycation of $\beta$-lactoglobulin," Food \& Function, vol. 6, no. 6, pp. 1841-1846, 2015.

[27] L. Cruz, I. Fernandes, M. Guimaraes, V. D. Freitas, and N. Mateus, "Enzymatic synthesis, structural characterization and antioxidant capacity assessment of a new lipophilic malvidin-3-glucoside-oleic acid conjugate," Food \& Function, vol. 7, no. 6, pp. 2754-2762, 2016.

[28] M. Yamamoto, H. Jokura, K. Hashizume et al., "Shimotoyodome, hesperidin metabolite hesperetin-7-O-glucuronide, but not hesperetin-3'-O-glucuronide, exerts hypotensive, vasodilatory, and anti-inflammatory activities," Food \& Function, vol. 4, no. 9, pp. 1346-1351, 2013.

[29] W. Hu, X. Wang, L. Wu et al., “Apigenin-7-O- $\beta$-D-glucuronide inhibits LPS-induced inflammation through the inactivation of AP-1 and MAPK signaling pathways in RAW 264.7 macrophages and protects mice against endotoxin shock," Food \& Function, vol. 7, no. 2, pp. 1002-1013, 2016.

[30] H. M. Li, J. K. Kim, J. M. Jang, S. O. Kwon, C. B. Cui, and S. S. Lim, "The inhibitory effect of Prunella vulgaris L. on aldose reductase and protein glycation," BioMed Research International, vol. 2012, Article ID 928159, 7 pages, 2012.

[31] S. Y. Mok and S. Y. Lee, "Identification of flavonoids and flavonoid rhamnosides from Rhododendron mucronulatum for. albiflorum and their inhibitory activities against aldose reductase," Food Chemistry, vol. 136, no. 2, pp. 969-974, 2013.

[32] M. Plioukas, C. Gabrieli, D. Lazari, and E. Kokkalou, "Phytochemical analysis with the antioxidant and aldose reductase inhibitory capacities of Tephrosia humilis aerial parts' extracts," Natural Product Research., vol. 30, no. 12, pp. 1366-1372, 2016.

[33] H. A. Jung, Y. S. Kim, and J. S. Choi, "Quantitative HPLC analysis of two key flavonoids and inhibitory activities against aldose reductase from different parts of the Korean thistle, Cirsium maackii," Food and Chemical Toxicology, vol. 47, no. 11, pp. 2790-2797, 2009.

[34] H. M. Li, S. H. Hwang, B. G. Kang, J. S. Hong, and S. S. Lim, "Inhibitory effects of Colocasia esculenta (L.) Schott constituents on aldose reductase," Molecules, vol. 19, no. 9, pp. 13212-13224, 2014.

[35] A. H. Jung, Y. J. Jung, N. Y. Yoon et al., "Inhibitory effects of Nelumbo nucifera leaves on rat lens aldose reductase, advanced glycation end products formation, and oxidative stress," Food and Chemical Toxicology, vol. 46, no. 12, pp. 3818-3826, 2008.

[36] H. N. Yoon, M. Y. Lee, J. K. Kim, H. W. Suh, and S. S. Lim, "Aldose reductase inhibitory compounds from Xanthium strumarium," Archives of Pharmacal Research, vol. 36, no. 9, pp. 1090-1095, 2013.

[37] H. Matsuda, T. Morikawa, I. Toguchida, and M. Yoshikawa, "Structural requirements of flavonoids and related compounds for aldose reductase inhibitory activity," Chemical and Pharmaceutical Bulletin, vol. 50, no. 6, pp. 788-795, 2002.

[38] M. Yoshikawa, T. Morikawa, T. Murakami, I. Toguchida, S. Harima, and H. Matsuda, "Medicinal flowers. I. Aldose reductase inhibitors and three new eudesmane-type sesquiterpenes, kikkanols $\mathrm{A}, \mathrm{B}$, and $\mathrm{C}$, from the flowers of 
Chrysanthemum indicum L," Chemical and Pharmaceutical Bulletin, vol. 47, no. 3, pp. 340-345, 1999.

[39] H. Matsuda, T. Morikawa, I. Toguchida, S. Harima, and M. Yoshikawa, "Medicinal flowers. VI. Absolute stereostructures of two new flavanone glycosides and a phenylbutanoid glycoside from the flowers of Chrysanthemum indicum L.: their inhibitory activities for rat lens aldose reductase," Chemical and Pharmaceutical Bulletin, vol. 50, no. 7, pp. 972-975, 2002.

[40] H. Matsuda, T. Wang, H. Managi, and M. Yoshikawa, "Structural requirements of flavonoids for inhibition of protein glycation and radical scavenging activities," Bioorganic \& Medicinal Chemistry, vol. 11, no. 24, pp. 5317-5323, 2003.

[41] S. H. Jung, J. M. Lee, H. J. Lee, C. Y. Kim, E. H. Lee, and B. H. Um, "Aldose reductase and advanced glycation end products inhibitory effect of Phyllostachys nigra," Biological and Pharmaceutical Bulletin, vol. 30, no. 8, pp. 1569-1572, 2007.

[42] J. K. Kim, Y. S. Lee, S. H. Kim, Y. S. Bae, and S. S. Lim, "Inhibition of aldose reductase by phenylethanoid glycoside isolated from the seeds of Paulownia coreana," Biological and Pharmaceutical Bulletin., vol. 34, no. 1, pp. 160-163, 2011.

[43] Y. S. Lee, J. K. Kim, Y. S. Bae, M. H. Won, I. J. Kang, and S. S. Lim, "Inhibitory effect of glucodistylin from the bark of Quercus acutissima on human recombinant aldose reductase and sorbitol accumulation," Archives of Pharmacal Research, vol. 34, no. 2, pp. 211-215, 2011.

[44] S. H. Kim, J. K. Kim, Y. S. Lee, Y. S. Bae, and S. S. Lim, "Inhibitory effect of quercetin-3-O- $\alpha$-L-rhamnopyranoside from Chamaecyparis obtusa on aldose reductase and sorbitol accumulation," Korean Journal of Medicinal Crop Science, vol. 18, no. 5, pp. 305-310, 2010. 


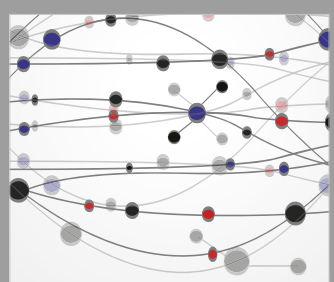

The Scientific World Journal
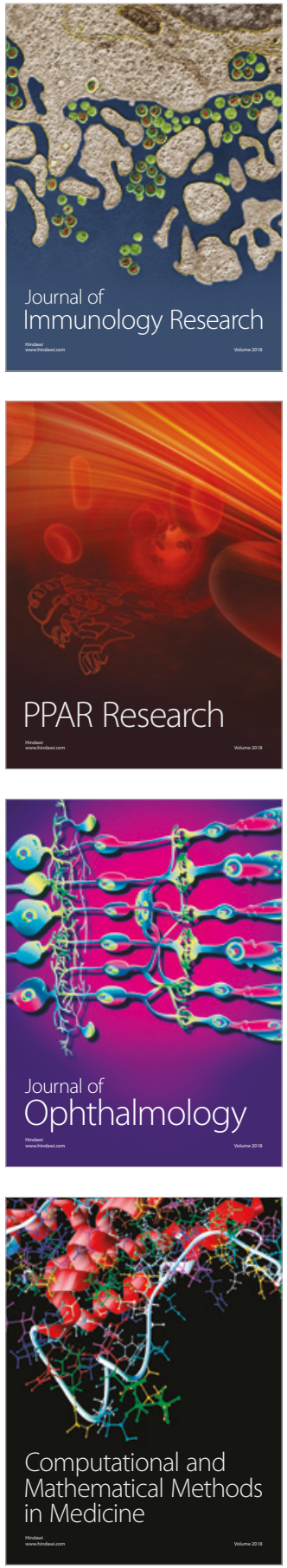

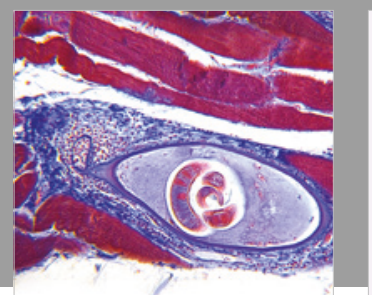

Gastroenterology Research and Practice

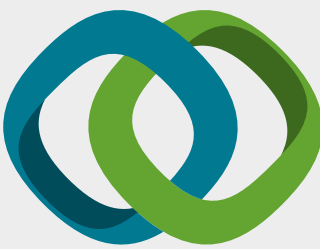

\section{Hindawi}

Submit your manuscripts at

www.hindawi.com
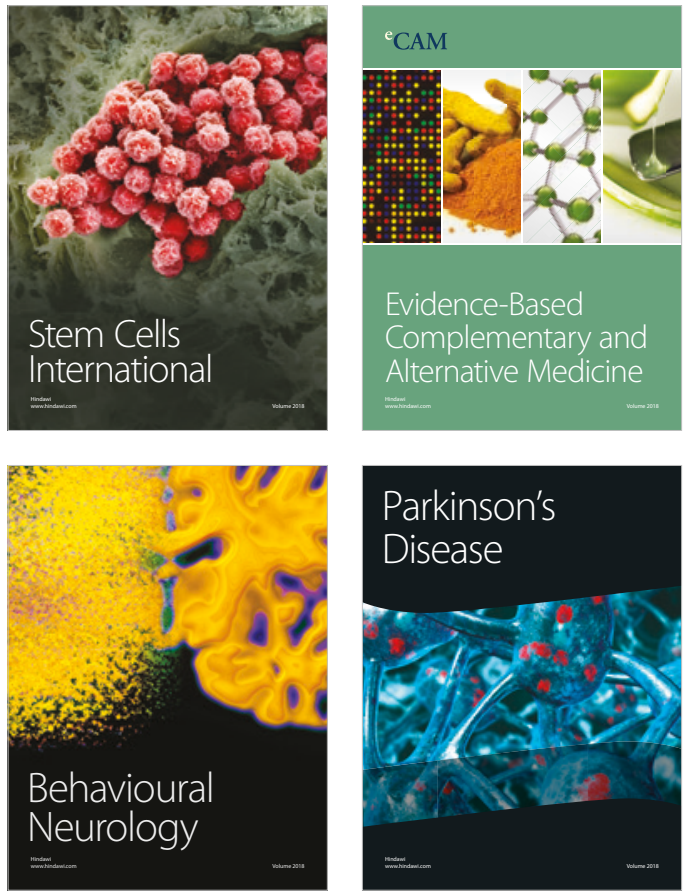

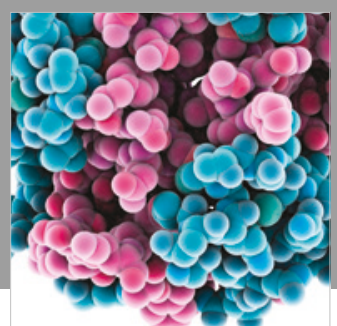

ournal of

Diabetes Research

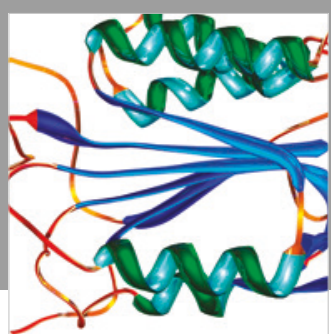

Disease Markers
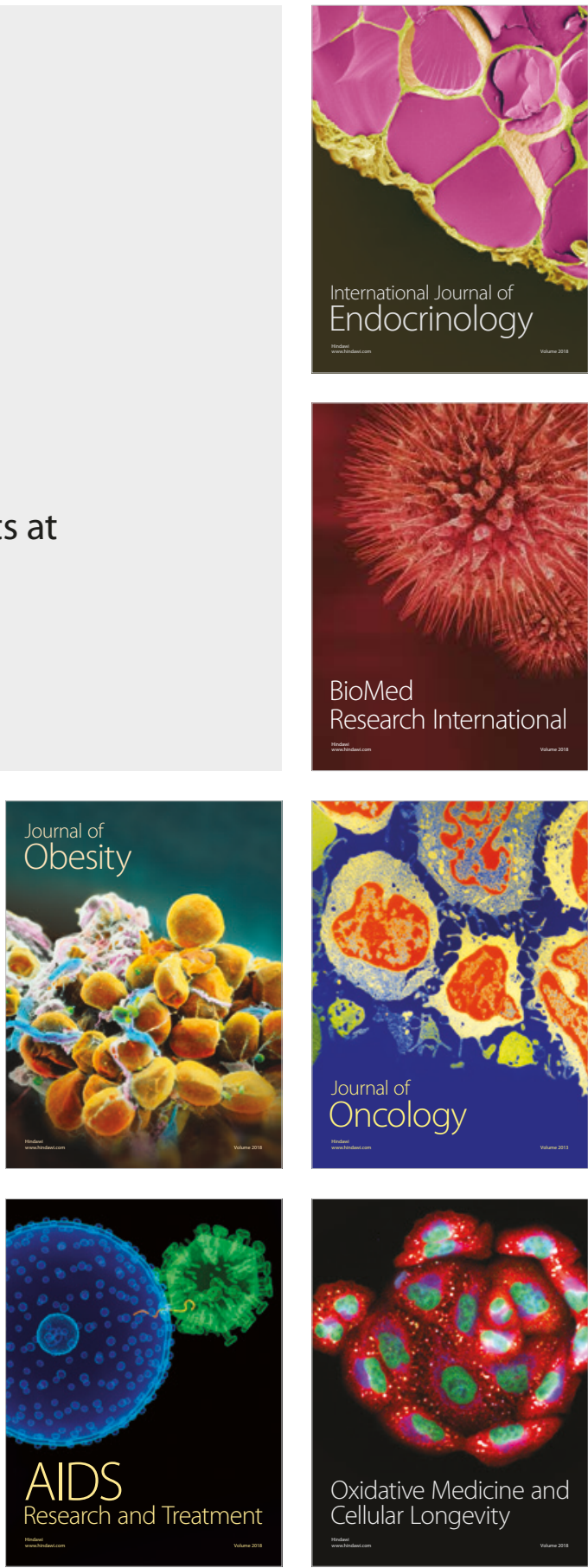